\title{
Developing a 3D-Printed Obese Model for Assessing Fit of Wearable Smart Garments
}

\author{
Mahendran BALASUBRAMANIAN*, Mary RUPPERT-STROESCU \\ Department of Design, Housing and Merchandising, Oklahoma State University, OK, USA
}

DOI: 10.15221/15.018 http://dx.doi.org/10.15221/15.018

\begin{abstract}
Clothing systems have been successfully used as a viable embedding media for several vital signals monitoring systems, making them smart garments. Two persistent issues with smart garments are improper fit and wearing discomfort. When collecting biometric data, precise sensor placement is extremely important, and when a garment is not comfortable, it risks not being used at all. Conventionally, fit is often assessed using fit models, either human subjects or static mannequins. Most companies determine fit model sizes that represent a median population, and the garments developed thus cannot be scaled directly to an obese body, which now represents a significant portion of the US population. In this study, fit models representing the obese population were selected from the CAESAR scan database for male and female and a male body form was additively manufactured using 3D printing technique. Digital scans of 185 obese male and 204 obese female from the CAESAR database were used to select the appropriate fit models. The bivariate distribution of chest and waist-front length measurements determined a representative subpopulation of 15 male cases and 14 female cases. Furthermore, sagittal cross section curvature analysis was performed on the cases to capture profile variations. Subsequently, the anthropometric distance variations among the cases for several key landmarks were measured to establish a range for sensor placement on the smart garment. From the 15 male cases, one subject was heuristically identified as the final fit model. The identified scan was preprocessed and torso region of the scan was extracted using the Polyworks V14 software. In order to make a life-like replication of the torso, two 3D printers were simultaneously used to print the model as 16 individual hollow slices. Subsequently, the slices were assembled together to produce the full scale obese-fit-model 3D form.
\end{abstract}

Keywords: Fit, 3D printing, fit-model, smart garments, obese

\section{Introduction}

Over the past two decades, clothing systems are gradually evolving from being passive apparel to what is called smart garments. These smart garments fall under the category of wearable electronics in which several monitoring devices are often embedded with the clothing so as to measure, monitor, and report human vital signals. Two long-standing issues with smart garments are wearing discomfort and improper fit. Both of these problems are often intertwined; a user is not likely to wear a garment that causes discomfort, and especially, improper fit leads to signal distortion and an unreliable system. In the case of improper fit, sensors contacts to the body may be disconnected, which eventually affect the quality of the signal by adding high and low frequency noise. If the characteristics of the noise are known a priori, we can eliminate them to restore the signal. However, with improper fit, the noise characteristics are non-stationary and are often hard to estimate, leading to unreliable and poor signal quality. Another most common but serious issue with improper fit is the motion artifact [1] that occurs with different dynamics of bodily motion.

Conventionally, fit is often assessed using fit models, either human subjects or static mannequins. Most companies determine fit model sizes that represent a median population and ignore outlying sizes, such as those necessary to fit obese persons, or those who possess excessive body fat accumulation that may impair health [2]. The obese category now represents a significant portion of the US population and those garments developed based on a median population cannot be effectively scaled directly to an obese body. When it comes to smart garments the tolerance of fit is particularly crucial. Especially, with special populations such as the obese group, employing standard fit models will not be very effective, thus indicating the need for new approaches to enabling smart garment development with accurate fit.

*Corresponding Author: mahendb@okstate.edu; Department of DHM, Oklahoma State University 
In this work, we present a novel methodology of generating fit models using additive manufacturing technique by using 3D body scan data of obese male and female populations. Through this analysis we also analyzed the curve propagation on the human torso to objectively inform optimal placement of sensors within the garment. The analyses were particularly streamed to be compatible with a garment design to detect sleep disorders by monitoring biometric parameters.

\section{Methodology}

\subsection{Fit Model selection}

The target populations for this study were obese males and females. The obese population is a distinct and rising demographic. For example, in 2014 over 600 million people over age 18 or $13 \%$ of the world's population were obese, a number that has more than doubled in the past 35 years [2]. Typical apparel industry fit model body forms are not readily available for the obese population. Therefore, in order to create a representative fit model for the obese population, we used a case selection approach based on key torso measurements derived from 3D body scanned data (CAESAR scan database [3]).

Generally, fit models are chosen based on a range of body measurements. These measurements can vary across apparel manufacturers [4,5]. General consensus indicates that this method lacks precision and efficiency even for regular garments, therefore there is a need for a different approach for smart garments. In this work, instead of the conventional fit model selection method, the case selection approach was used to determine the sub-population of the male and female obese populations. From the entire population, subjects were classified as obese based on their body-mass index (BMI). Subjects with BMI $\geq 30$ were considered obese [6]. All the subjects used in this study were American civilians. For the male population, 185 subjects were chosen and for the female population, 204 subjects were selected. The 3D data used in the analyses were processed using the Polyworks V14 software. Two key measurements were extracted from these 3D data: the chest/bust circumference and the waist-front length. The definitions for these measurements are given in Table1 , and 14/15 cases per gender were chosen based on the bivariate distributions of the chosen measurements (see Figure-1).

Table 1. Measurement locations and definitions

\begin{tabular}{|l|l|l|}
\hline \multicolumn{1}{|c|}{} & \multicolumn{1}{|c|}{ Measurement } & \multicolumn{1}{|c|}{ Definition } \\
\cline { 2 - 3 } & Chest/Bust circumference & $\begin{array}{l}\text { Circumference of the torso } \\
\text { measured at nipple level }\end{array}$ \\
\cline { 2 - 3 } & Waist-front length & $\begin{array}{l}\text { Surface distance from a } \\
\text { point on the front neck base } \\
\text { (in the midsagittal plane) is } \\
\text { measured to the anterior } \\
\text { preferred waist [7]. }\end{array}$ \\
\hline
\end{tabular}

To assess homogeneity and ensure minimal variance among the chosen subjects, sagittal cross sections were extracted at the suprasternale (STR) landmark and subjectively evaluated (see Figure2). A plane was drawn that is vertical to the floor in the sagittal axis and crosses the STR. The scans from all the subjects, grouped by gender, were aligned at the STR before extracting the vertical measurements. The variance in the vertical distance between the STR to Thelion and STR to $10^{\text {th }}$ rib, the 3D torso was estimated using the 3D torso scan. This is particularly important when developing smart garments for healthcare purposes because, for example, in cases where a respiratory band needs to be embedded in the garment, the variance in the vertical distance between the STR to Thelion (chest/bust) point becomes important for a reliable signal measurement. Similarly, to place the reference electrodes for the ECGs, the variance in the vertical distance between the STR marker and the $10^{\text {th }}$ rib becomes a measure of interest. 


\subsection{D printing}

Printing in $3 D$ is an additive manufacturing technique used widely for rapid prototyping. In this work, a scan of the male subject representing the 15 cases was chosen heuristically and 3D printed. We used the fused deposit modelling approach and printed the torso of the fit model in full scale using polylactic acid polymer (PLA). The printers used for the purpose were (a) Cubex (print volume: $10.8^{*} 10.45^{*} 9.5 \mathrm{in}$ ) and (b) Ultimaker (Print volume: $9 * 8.8^{*} 8 \mathrm{in}$ ). Because the 3D torso was occupying a much larger volume compared to the printers' capacity, the full-scale digital torso was sliced into 16 non-overlapping individual pieces, and printed as hollow structure (so as to minimize the polymer consumption). The pieces were then assembled together forming the full-scale obese-male-fit model. The same sequence is applicable for the female model.

\section{Results}

Figure 1 shows the bivariate distribution of chest and waist-front length for both the genders. The red colored markers indicate the male and female subjects selected as cases. The sagittal cross section of the 15 male cases and 14 female cases are shown in Figure 2. The cross-section analysis of the cases for both male and female subjects shows that the profile curvatures are more or less similar with all the cases within the gender group. The scan alignment and the measurement variations are shown in Figure 3. The vertical distance measured between (a) STR and Thelion, and (b) STR and $10^{\text {th }}$ rib are shown in Table 2. The plot in Figure 4 shows the mean and deviations of these two measurements. The torso of the male subject chosen to be 3D printed is shown in Figure 5 on the left. The 3D printed model was evaluated for its accuracy. To estimate the deviances between the printed prototype and the digital representation, the 3D printed prototype was scanned using human solutions 3D body scanner and aligned with the original digital model. The middle image in Figure 5 shows the accuracy of the printed model. The red color torso is the scan of human and the gray color mesh is the $3 \mathrm{D}$ scan of the printed model. It shows very minimal error in the dimensions. The final printed model is shown in Figure 5 on the right.
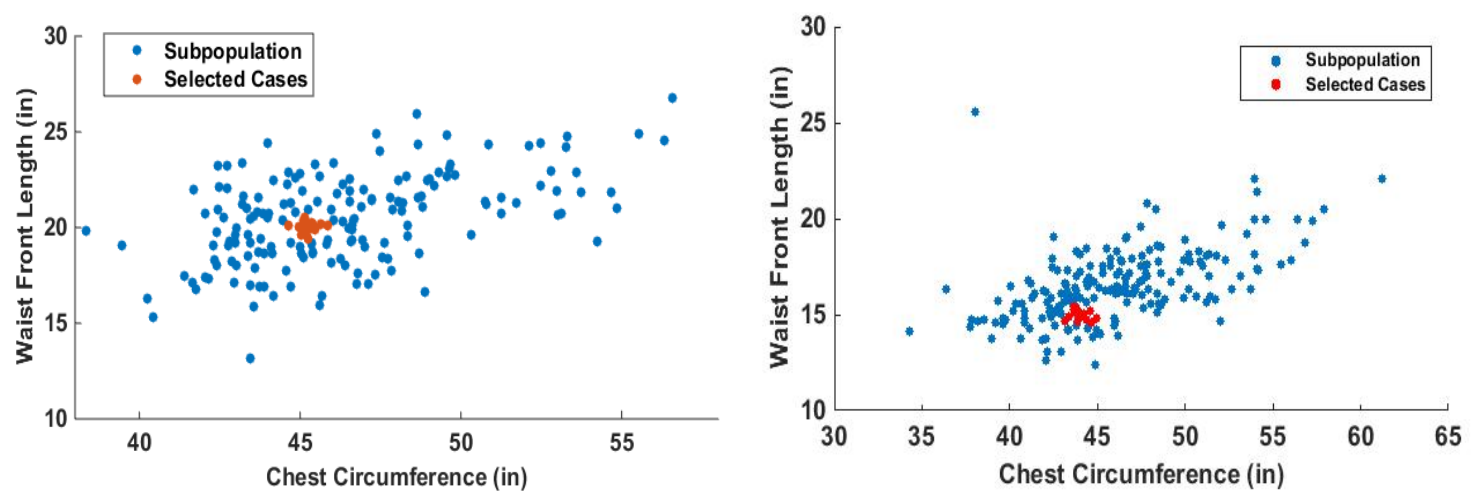

Fig.1. Bivariate distribution of Chest circumference and Waist front length for Male (left) and Female (right).
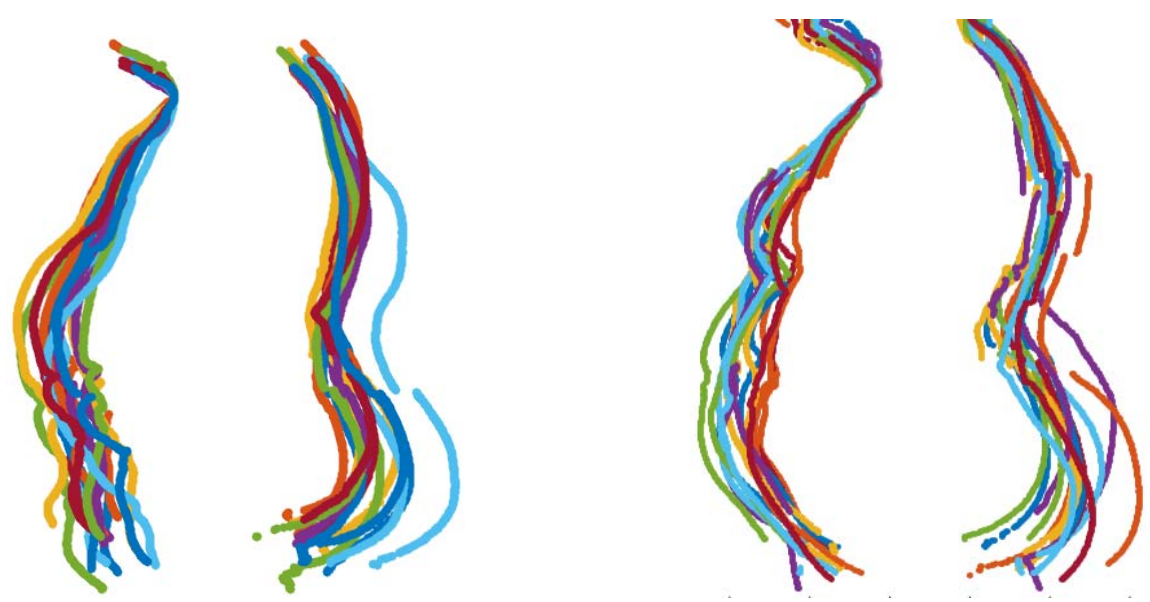

Fig. 2. Sagittal cross-section of the cases - Male (left) and Female (right) 
Table 2. Summary of the vertical distances between landmarks

\begin{tabular}{|l|l|c|c|}
\hline Gender & Parameters & Suprasternale to Thelion $\mathbf{( c m )}$ & Suprasternale to 10th rib $\mathbf{( c m})$ \\
\hline \multirow{4}{*}{ Male } & Min & 14.19 & 28.07 \\
\cline { 2 - 4 } & Max & 18.96 & 35.98 \\
\cline { 2 - 4 } & Average & 16.81 & 31.86 \\
\hline \multirow{3}{*}{ Female } & Min & 16.32 & 24.58 \\
\cline { 2 - 4 } & Max & 22.30 & 31.25 \\
\cline { 2 - 4 } & Average & 19.83 & 29.48 \\
\hline
\end{tabular}

\section{Discussions}

Measuring vital signals using wearable technologies is generally being done using standalone devices mounted on to the human body. However, when multiple signals are needed to be measured, mounting them individually on a subject's body repeatedly becomes cumbersome. Alternatively, these sensing devices can be attached to a smart garment such that the relative positioning of multiple sensors can be pre-determined and embedded into the clothing system. In order to ensure aligning of the sensor with the needed body landmarks, such as, (i) the ECG reference electrode goes to the $10^{\text {th }}$ rib, (ii) the respiration sensor positioning in reference to the Thelion landmark, etc, the representative variance of these landmarks need to be known a priori. The current work provides two distinct sets of information for garment designing, (a) creating a 3D form representing a special population to emulate sensor placements and (b) estimating variances in the landmarks on human body. In real-world situation, the variances in the position of such landmarks need to be taken in to account while designing the patterns for smart garments. The $3 \mathrm{D}$ scan data of the male subjects show that the distance between the STR and the Thelion spans over a range of $4.77 \mathrm{~cm}$, and for female it was 5.98 $\mathrm{cm}$. Likewise, the distance between the STR and the $10^{\text {th }}$ rib had a range of $7.9 \mathrm{~cm}$ for the male subjects and $6.66 \mathrm{~cm}$ for the female subjects. These tolerances are to be considered during the pattern making to ensure proper signal acquisition. Anthropometrically, the STR to Thelion distance is higher for female than male cases and the STR to $10^{\text {th }}$ distance is higher for male than female cases. These things are to be noted, if the smart garment is planned to be designed as a unisex product.

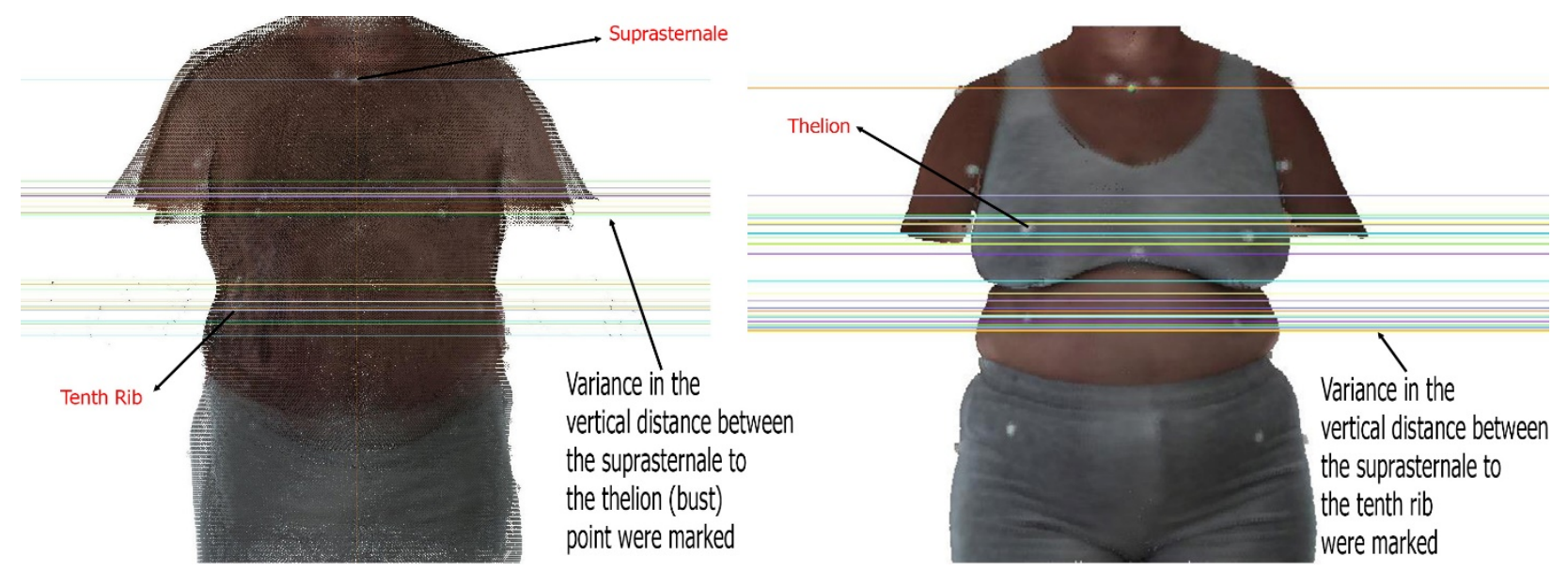

Fig. 3. 3D scans aligned at suprasternale (STR) and the variance measured from STR to Thelion and STR to $10^{\text {th }}$ Rib. Multiple lines at a landmark shows for individual subject measurements

The 3D printed male torso served as the body form for initial fit assessment of the vest as well as for sensors placements. The fit variations due to encumbrance of data acquisition board, battery and other components were identified using the 3D form. 

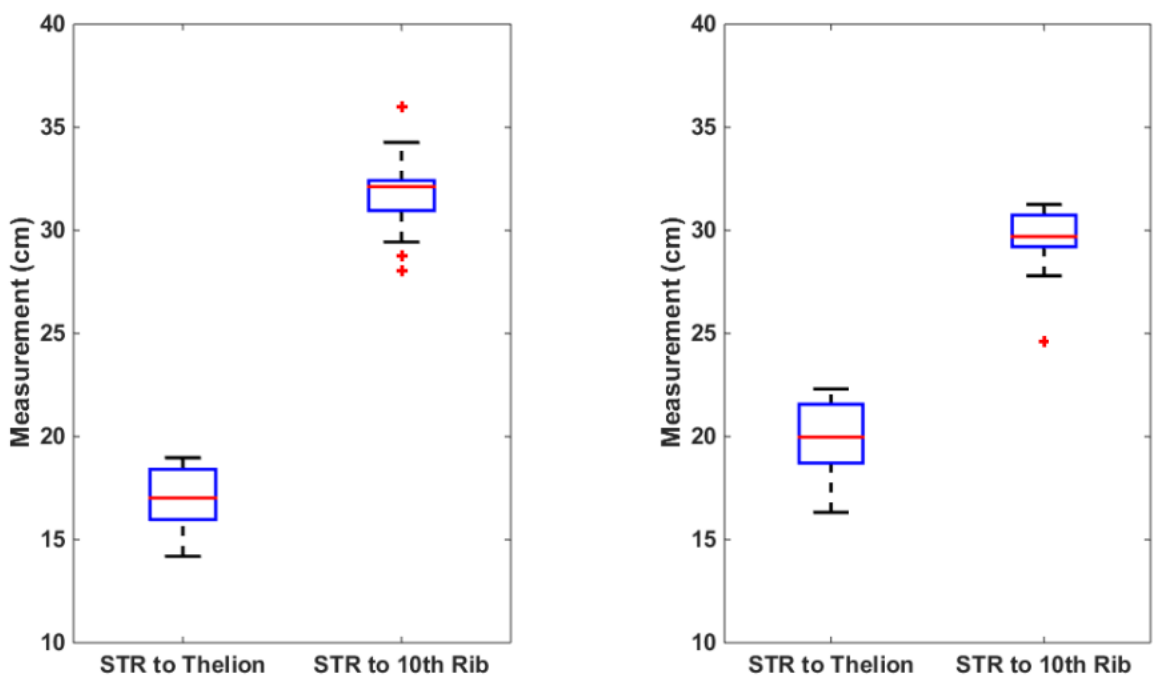

Fig. 4. Mean and deviation of the vertical distances measured (Left) Male subjects and (Right) Female subjects

The printed torso was rescanned using a 3D body scanner to evaluate its accuracy compared to the original $3 \mathrm{D}$ data. The scanned version of the $3 \mathrm{D}$ printed torso was then aligned with the original digital scan of the subject to estimate the error of accuracy. Minimal errors were found in the circumference dimensions; however, they are not significant and were well within the tolerance limits.
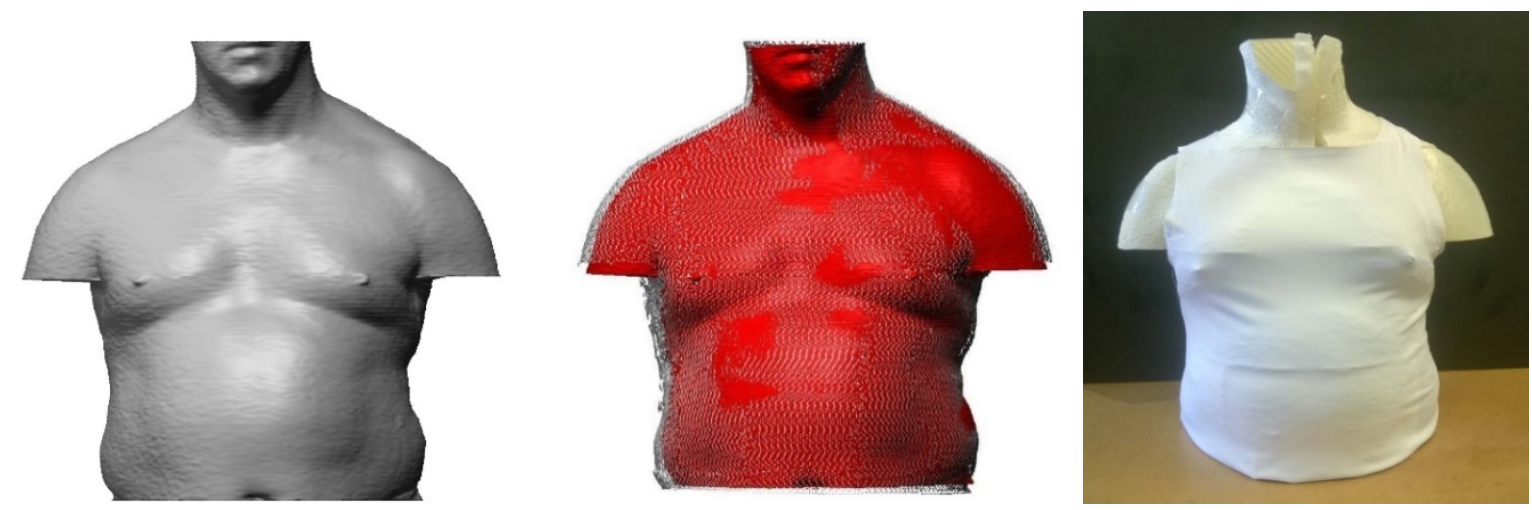

Fig. 5. (Left) 3D Scan of the selected subject for prototyping; (Middle) Comparison of the digital scans of the original subject (red) and the 3D printed prototype (grey); (Right) 3D printed obese fit model in full scale

\section{Conclusion}

Suitable obese fit models were selected using the case selection approach and a prototype was printed using the 3D printing technique. The variances in the measurements were quantified for optimal sensor placement for respiratory band and ECG sensors for each of the male and female groups. This 3D printed model served as a physical fit assessment model and an evaluation platform for mounting data acquisition modules. Implications of this research can be applied to not only smart garment development, to improve fit and comfort, and optimal placement of sensors, but will also help determine the dimensions of the sensing components themselves, such as electrodes and respiratory bands.

\section{Acknowledgment}

The authors would like to thank Dr. Kathleen Robinette for sharing the CAESAR scans for this study. 


\section{References}

1. W. Frank, W. Roth, and M. Sackner. "The LifeShirt An Advanced System for Ambulatory Measurement of Respiratory and Cardiac Function." In Behavior Modification, Vol. 27. No.5, 2003, pp. 671-691.

2. Obesity and overweight, http://www.who.int/mediacentre/factsheets/fs311/en/, accessed Aug, 2015

3. WEAR: World Engineering Anthropometry Resource, http://www.bodysizeshape.com/, accessed Feb, 2015.

4. J. Workman. "Body measurement specifications for fit models as a factor in clothing size variation." In Clothing and Textiles Research Journal, Vol. 10, No.1, 1991, pp. 31-36.

5. J. Workman and E. Lentz. "Measurement specifications for manufacturers' prototype bodies." in Clothing and Textiles Research Journal, Vol.18, No.4, 2000, pp. 251-259.

6. Overweight and Obesity, http://www.cdc.gov/obesity/adult/defining.html, accessed Feb, 2015

7. S. Blackwell, et al. (2002). Civilian American and European Surface Anthropometry Resource (CAESAR), Final Report. Vol. 2. Wright-Patterson AFB, Ohio: Air Force Research Laboratory, Human Effectiveness Directorate, Crew System Interface Division. 